\section{Histochemische Untersuchungen über die Glucose-6-Phosphatase in der Rattenleber während der Kanzerisierung durch Nitrosamine}

Von W. Gössner und H. Friedrich-Freksa

Pathologisches Institut der Universität Tübingen und Max-Planck-Institut für Virusforschung, Abteilung für physikalische Biologie, Tübingen

(Z. Naturforschg. 19 b, 862-863 [1964] ; eingegangen am 8. Juni 1964)

Die Cyto- und Histogenese des durch Diäthylnitrosamin entstehenden Leberkarzinoms der Ratte ${ }^{1}$ ist in einer Reihe von Arbeiten lichtmikroskopisch 2, 3, elektronenmikroskopisch ${ }^{4}$, cytophotometrisch ${ }^{5}$ und autoradiographisch ${ }^{6}$ ausführlich dargestellt worden. Das Fehlen entsprechender enzymhistochemischer Untersuchungen veranlaßte uns, das Verhalten einer Reihe histochemisch nachweisbarer Enzyme in der Leber im Verlauf der Karzinogenese zu untersuchen.

In der vorliegenden Mitteilung soll über die Befunde beim histochemischen Nachweis der Glucose-6-Phosphatase (G6P) berichtet werden. In den bisher vorliegenden histochemischen Arbeiten bei experimentell erzeugten Lebertumoren (nach Dimethylaminoazobenzol, 3-Methyl-DMAB, Äthionin) sind über das Verhalten dieses Enzyms noch keine Angaben zu finden.

\section{Methoden}

Die Untersuchungen wurden an weiblichen Ratten des Stammes Sprague-Dawley, die Diäthylnitrosamin (DÄNA) in einer täglichen Dosierung von $5 \mathrm{mg} / \mathrm{kg}$ Körpergewicht mit einer Schlundsonde und Nitrosomorpholin in einer Tagesdosis von etwa $8 \mathrm{mg} / \mathrm{kg}$ mit dem Trinkwasser erhielten, durchgeführt. Die Tiere wurden zunächst in täglichen, später in wöchentlichen Abständen nach Beginn der Behandlung getötet. Von der Leber wurden Kryostatschnitte hergestellt und diese sowohl enzymhistochemisch, als auch mit histologischen Färbemethoden bearbeitet.

Der Nachweis der G6P erfolgte nach der Methode von $\mathrm{W}_{\text {Achstern }}$ und Meisel ${ }^{10}$. Die G6P wurde erstmals von Chiquorne ${ }^{11}$ mit Hilfe einer Metallsalzmethode (in Gegenwart von $\mathrm{Pb}^{2 \oplus}$ ) in unfixierten Gewebsschnitten von Leber, Niere und Darm nachgewiesen. Das ursprüngliche Verfahren wurde durch das von $\mathrm{W}$ a $\mathrm{ch}$ -

1 D. Schmähl, R. Preussmann u. H. Hamperl, Naturwissenschaften 47, 89 [1960].

2 C. Thomas, Z. Krebsforsch. 64, 224 [1961].

3 E. Grundmann u. H. Sieburg, Beitr. pathol. Anatom. allg. Pathol. 126, 57 [1962].

4 E. Mölbert, K. Hill u. F. Büchner, Beitr. pathol. Anatom. allg. Pathol. 126, 218 [1962].

5 H. P. Новiк u. E. Grundmann, Beitr. pathol. Anatom. allg. Pathol. 127, 25 [1962].

6 W. Oehlert u. J. Hartje, Beitr. pathol. Anatom. allg. Pathol. 128, 376 [1963].

7 M. Wachstein u. E. Meisel, J. Histochem. Cytochem. [Baltimore] 7, 189 [1959].

8 H. Heinlein, G. Hübner, K. J. Lennartz u. G. Rudolph, Klin. Wschr. 40, 121 [1962].

9 H. P. Morris, Progr. exp. Tumor Res. 3, 370 [1963]. s te in und $\mathrm{M}$ e i s e l modifizierte Inkubationsmedium verbessert.

Mit dieser Methode gelang auch der Nachweis von G6P in den B-Zellen der Pankreasinseln ${ }^{12,}{ }^{13}$, im Nebenhoden der Maus ${ }^{14}$ und in Ganglienzellen ${ }^{15}$. Gegenüber der unspezifischen sauren Phosphatase läßt sich im histochemischen Präparat die G6P durch ihre Formalinempfindlichkeit, das abweichende $p_{\mathrm{H}}-\mathrm{Optimum}$, das Verhalten gegenüber Hemmstoffen und die unterschiedliche zelluläre und gewebliche Lokalisation sicher abgrenzen.

In letzter Zeit wurde über die elektronenmikroskopische Darstellung der G6P in der Leberzelle mit der W a chstein-M e is el-Methode berichtet. Dabei stellte sich das Reaktionsprodukt an den glatten und rauhen Elementen des endoplasmatischen Retikulums und an der äußeren Kernmembran dar ${ }^{16}$. Dies steht mit biochemischen Befunden in Einklang, wonach die G6P in der Mikrosomenfraktion enthalten ist ${ }^{17}$.

\section{Ergebnisse}

An der normalen Rattenleber konnten wir bestätigen, daß die G6P ausschließlich im Cytoplasma der Leberzellen lokalisiert ist und die Ablagerung des Reaktionsproduktes etwa der Verteilung der cytoplasmatischen basophilen Schollen entspricht. Im allgemeinen ist die Aktivität in der Läppchenperipherie stärker als im Läppchenzentrum. Im Hungerzustand kommt es zu einer deutlichen Verstärkung der Enzymaktivität.

In den durch DÄNA und Nitrosomorpholin erzeugten Leberkarzinomen konnten wir histochemisch keine G6P nachweisen. Dies steht mit der Tatsache in Übereinstimmung, daß auch in biochemischen Untersuchungen bei einer Reihe von Hepatomen bzw. Leberkarzinomen ein Fehlen dieses Enzyms festgestellt wurde ${ }^{18}$. Es trifft dies allerdings nicht für alle in der experimentellen Tumorforschung bekannten Lebergeschwülste $\mathrm{zu}^{9,19}$.

Im Hinblick auf diesen Enzymdefekt im NitrosaminKrebs der Rattenleber sind die Befunde, die wir im Laufe der karzinogenen Behandlung erheben konnten, von besonderem Interesse. Es treten bereits sechs Wochen nach Beginn der Behandlung und im Laufe der nächsten Wochen zunehmend (nach etwa 200 bis

10 M. Wachstein u. E. Meisel, J. Histochem. Cytochem. [Baltimore] 4, 592 [1956].

11 A. D. Chiquorne, J. Histochem. Cytochem. [Baltimore] 1, 429 [1953] ; 3, 471 [1955].

12 S. S. Lazarus, Proc. Soc. exp. Biol. Med. 101, 819 [1959].

13 W. Gössner, Fortschritte der Diabetesforschung, G. Thieme, Stuttgart 1963, S. 140.

14 J. M. Allen, J. Histochem. Cytochem. [Baltimore] 9, 681 [1961].

15 H. B. Tewari u. G. H. Bourne, J. Histochem. Cytochem. [Baltimore] 11, 121 [1963].

16 L. W. Tice u. R. J. Barrnett, J. Histochem. Cytochem. [Baltimore] 10, 754 [1962].

17 C. De Duve, B. C. Pressman, R. Gianetto, R. Mattiaux u. F. Appelmans, Biochem. J. 60, 604 [1955].

18 G. Weber u. A. Cantaro, Cancer Res. 15, 105 [1955].

19 H. C. Piтот, Federat. Proc. 21, 1124 [1962]. 
$300 \mathrm{mg} / \mathrm{kg}$ DÄNA) zunächst kleinere Leberzellnester, später größere Zellinseln auf, in denen keine G6P nachweisbar ist. Dagegen zeigt das übrige Lebergewebe eine kräftige, in der Intensität lediglich etwas unterschiedliche Enzymreaktion. Diese enzymfreien Zellinseln sind von unterschiedlicher Größe, im Schnitt ungleichmäßig verteilt, häufig aber im Läppchenzentrum und scharf gegenüber den angrenzenden enzymhaltigen Leberzellen abgesetzt $\left(\mathrm{Abb} .1^{*}\right)$. Die Leberzellen im Bereich dieser Inseln zeigen keinerlei Kernregressionen, ihr Cytoplasma ist zunächst von vakuolärer, in späteren Stadien von deutlich basophiler Beschaffenheit.

Die vorliegenden Befunde zeigen also, da $\beta$ ein beim experimentellen Leberkarzinom der Ratte nach Behandlung mit DÄNA und Nitrosomorpholin histochemisch nachweisbarer Enzymdefekt, nämlich der Verlust der mikrosomalen G6P, bereits im präkanzerösen

* Abb. 1 s. Tafel S. 852 a.

20 E. WeIler, Z. Naturforschg. 11 b, 31 [1956].

21 K. Porter u. C. Bruni, Cancer Res. 19, 997 [1959].

\section{Untersuchungen zur Wirkung negativer atmosphärischer Kleinionen auf die Mutabilität bei Drosophila melanogaster}

\section{Von E. Magdon, H. Czihąz und G. Winterfeld}

Robert-Rössle-Klinik (Direktor Professor Dr. H. Gummer) und Institut für Biophysik (Direktor Professor Dr. H. LANGE) der Deutschen Akademie der Wissenschaften, Berlin-Buch

(Z. Naturforschg. 19 b, 863-864 [1964] ; eingegangen am 20. Juni 1964)

In einer großen Zahl von Arbeiten ist über die biclogische Wirkung von künstlich erzeugten atmosphärischen Kleinionen berichtet worden ${ }^{1-5}$. In letzter Zeit ist versucht worden, den Mechanismus der biologischen Luftionenwirkung durch Radikalbildung zu erklären ${ }^{6}$. Von $\mathrm{KIngDoN}^{7}$ wurde dabei die biologische Wirkung von Luftionen ähnlich der indirekten Wirkung ionisierender Strahlung interpretiert. Entsprechend dieser Wirkungsweise wurde die Möglichkeit einer der Wirkung geringer Strahlendosen entsprechenden Induktion von Neoplasien des Bronchialtraktes diskutiert. Aus den in der oben genannten Literatur gegebenen Hinweisen ergab sich die Frage, ob negative Luftionen eine der Wirkung ionisierender Strahlung vergleichbare Auslösung von Mutationen verursachen können.

Zur Prüfung dieser Frage wurden adulte, 1-3 Tage alte Männchen von Drosophila melanogaster, Stamm Berlin wild, $16 \mathrm{Stdn}$. lang negativen Luftionen ausgesetzt, die mit einem Tritium-Ionen-Generator erzeugt wurden. Die Trennung der beiden Ionenpolaritäten er-

1 L. L. Vasil'ev, Theorie und Praxis der Behandlung mit ionisierter Luft, Leningrad 1953.

2 A. A. Minkr, Die Ionisation der Luft und ihre hygienische Bedeutung. Medgiz. 1958.

3 F. G. Portnov, Die Luftionen und ihre therapeutische Anwendung. Riga 1961.

4. J. H. Konnbluch, Archives of Medial Hydrology [Italy] 21, 1 [1961].
Stadium im Bereich umschriebener Leberzellinseln in Erscheinung tritt. Eine Korrelation dieser Beobachtung mit anderen bei der Entstehung des experimentellen Leberkarzinoms bereits bekannten Befunden, nämlich

1. dem histo-serologischen Nachweis umschriebener Zellnester mit einem Schwund von leberspezifischem, mikrosomalen Antigen ${ }^{20}$,

2. den autoradiographisch nachweisbaren Zellgruppen mit verstärkter RNS-Synthese ${ }^{6}$,

3. den glykogenfreien Zellinseln ${ }^{3}$,

4. den elektronenmikroskopisch festgestellten Veränderungen am endoplasmatischen Retikulum ${ }^{4,21,22}$

und die Frage, ob auch ein entsprechender Verlust anderer Mikrosomenenzyme (Hydroxylasen, besonders Fremdstoffhydroxylasen) erfolgt, muß weiteren Untersuchungen vorbehalten bleiben.

22 P. Emmelot u. E. L. Benedetti, J. biophysic. biochem. Cytol. 7, 393 [1960].

folgte durch Anlegung einer negativen Gleichspannung von $2000 \mathrm{~V}$ an der Elektrode hinter dem Tritium-Präparat (mit Tritium imprägnierte Zirkoniumscheibe). Im Abstand von ca. $3 \mathrm{~cm}$ vom Tritium-Präparat, in dem sich das Versuchsobjekt befand, wurde ein Ionenstrom von $10^{10}$ Ionen $/ \mathrm{cm}^{2} \cdot \mathrm{sec}$ gemessen.

Mittels des M-5-Verfahrens ${ }^{8}$ wurde die Auslösung rezessiver geschlechtsgebundener Letalfaktoren geprüft und mit einem gleichzeitig durchgeführten Kontrollversuch verglichen. Es wurden stets nur Einzelkreuzungen durchgeführt. Zur Untersuchung gelangten reife Spermien (Serie I, 0-3 Tage nach Exposition) sowie Spermatogonien (Serie VI, 15-18 Tage nach Exposition). Im Zeitintervall zwischen den beiden Bruten wurden die Männchen im Abstand von 3 Tagen mit neuen unbefruchteten Weibchen gekreuzt, um eine kontinuierliche Spermienabgabe zu garantieren.

Obwohl in beiden untersuchten Serien eine größere Häufigkeit rezessiver Letalfaktoren nach der Exposition mit negativen Ionen beobachtet wurde, ist der Unterschied zum Kontrollversuch in keinem Fall signifikant. Die Fehlerberechnung wurde sowohl nach dem $X^{2}$.Test ${ }^{9}$ als auch nach dem Verfahren von Stevens ${ }^{10}$ durchgeführt.

Die Erhöhung der Anzahl rezessiver Letalfaktoren in beiden untersuchten Serien gegenüber den Kontrollen legt die Vermutung nahe, daß in weiteren Versuchen mit einer größeren Anzahl untersuchter Chromosomen durch die Verwendung von Ionen-Generatoren mit größerer Ionenausbeute eine signifikante Steigerung der natürlichen Mutationsrate möglich sein könnte.

5 M. Knoll, J. Eichmeier u. K. W. Schön, Elektromedizin 7, 209 [1962].

B A. P. Krueger, Journal of the Albert Einstein Medical Center 8, 79 [1960].

7 K. H. Kingdon, Nature [London] 189, 180 [1961].

8 W. P. Spencer and C. Stern, Genetics 33, 43 [1948].

- E. Weber, Grundriß der biologischen Statistik. GustavFischer-Verlag, Jena 1961, 386.

10 W. L. Stevens, J. Genetics 43, 301 [1942]. 